\title{
Motor planning modulates neural activity patterns in early human auditory cortex
}

Daniel J. Gale1, Corson N. Areshenkoff ${ }^{1,2}$, Claire Honda², Ingrid S. Johnsrude ${ }^{5,6,7}$, J. Randall Flanagan ${ }^{1,2}$, \& Jason P. Gallivan*1,2,3

${ }^{1}$ Centre for Neuroscience Studies, ${ }^{2}$ Department of Psychology, and ${ }^{3}$ Department of Biomedical and Molecular Sciences, Queen's University, Kingston, Ontario, Canada. ${ }^{5}$ Department of Psychology, School of Communication Sciences and Disorders ${ }^{6}$, and ${ }^{7}$ Brain and Mind Institute, University of Western Ontario

Abbreviated Title: Motor planning modulates auditory cortex

Number of Pages: 39

Number of Figures: 4

Number of Supplemental Figures: 5

${ }^{*}$ Correspondence should be addressed to:

Jason Gallivan

Centre for Neuroscience Studies

Queen's University

gallivan@queensu.ca 


\section{Abstract}

It is well established that movement planning recruits motor-related cortical brain areas in preparation for the forthcoming action. Given that an integral component of the control of action is the processing of sensory information throughout movement, we predicted that movement planning also selectively modulates early sensory cortical areas in anticipation of the upcoming behaviour. To test this hypothesis, we performed two human functional MRI studies involving delayed movement tasks and focused on pre-movement neural activity patterns in early auditory cortex, given its direct connections to the motor system and evidence that motor cortex modulates auditory activity during subsequent movement execution. We show that effector-specific information (i.e., movements of the left vs. right hand in Experiment 1, and movements of the hand vs. eye in Experiment 2) can be decoded, well before movement, from neural activity patterns in early auditory cortex. In Experiment 1, we find that the decoding of this motor-related information occurs in a separate subregion of auditory cortex than the decoding of the sensory-related information used to instruct the action. In Experiment 2, we demonstrate that the representation of effector-specific information in auditory cortex, like that in the motor system, is contralateral in nature, and that this representation is present even when movements are cued visually, instead of auditorily. Together, these findings suggest that action planning, in addition to preparing the motor system for movement, involves the modulation of primary sensory areas, presumably readying them for the processing of sensory information that arises during the unfolding action.

\section{Keywords}

Motor, Planning, Auditory Cortex, Object manipulation, Action 


\section{INTRODUCTION}

Most theories of motor control distinguish between the planning of a movement and its subsequent execution. Research examining the neural basis of movement planning has commonly used delayed movement tasks-in which instructions about what movement to perform are separated in time from the instruction to initiate that movement-and has focused on delay period activity in motor-related brain areas. The main focus of this work has been on understanding how planning-related neural activity relates to features of the forthcoming movement to be executed e.g., 1,2, and more recently, how the dynamics of these neural activity patterns ultimately shape outgoing motor commands ${ }^{3}$. However, in addition to generating appropriate muscle commands, a critical component of skilled action is the prediction of the sensory consequences of motor commands ${ }^{4}$. For example, the sensorimotor control of object manipulation tasks involves predicting the sensory signals associated with contact events, which define subgoals of the task ${ }^{5}$. Importantly, these signals can occur in multiple sensory modalities, including tactile, proprioceptive, visual, and auditory ${ }^{6}$. By comparing the predicted to actual sensory outcomes, the brain can monitor task progression, detect performance errors, and quickly launch appropriate, task-protective corrective actions as needed. For example, when lifting an object that is heavier than expected, anticipated tactile events, associated with lift-off, fail to occur at the expected time, triggering a corrective response. Likewise, similar compensatory behaviour has also been shown to occur when anticipated auditory events fail to occur at the predicted time ${ }^{7}$. Sensory prediction is also critical in sensory cancellation, such as the attenuation of predictable sensory events that arise as a consequence of movement. Such attenuation is thought to allow the brain to disambiguate sensory events due to movement from events due to external sources ${ }^{8}$.

Given the functional importance of predicting task-specific sensory consequences, we hypothesized that action planning, in addition to preparing motor areas for execution, involves the preparation of primary sensory areas for processing task-specific sensory 
signals. Given that these sensory signals will depend on the precise action being performed, this hypothesis predicts that neural activity in early sensory areas will represent specific motor-related information prior to the movement being executed, during action preparation. One sensory system that is particularly well suited to directly testing this hypothesis is the auditory cortex. The mammalian auditory system exhibits an extensive, highly interconnected web of feedback projections, providing it with access to the output of information processing across multiple distributed brain areas ${ }^{9}$. To date, this feedback architecture has been mainly implicated in supporting auditory attention and working memory processes 10,11. However, recent work has also demonstrated that the auditory cortex also receives significant direct projections from ipsilateral motor cortex ${ }^{12-14}$. Consistent with this coupling between the motor and auditory systems, recent studies in both humans and rodents have shown that auditory cortex is functionally modulated by top-down motor inputs during movement execution 14,15. While the focus of this prior work has been on the real-time attenuation, during movement execution, of the predictable auditory consequences of action, it did not selectively focus on the movement planning process itself, or the broader function of the motor system in modulating early auditory activity in preparation for forthcoming sensorimotor control.

Here we show, using functional MRI and two separate delayed movement experiments involving object manipulation, that the movement effector to be used in an upcoming action can be decoded from delay period activity in early auditory cortex. We demonstrate that this pre-movement decoding in the auditory cortex is automatic in nature, independent of the sensory input used to cue movement, and occurs contralateral to the motor effector being used. Beyond real-time sensory attenuation, these findings suggest that, during movement preparation, the motor system selectively changes the neural state of early auditory cortex in accordance with the specific motor action being prepared, likely readying it for the processing of sensory information that will arise during subsequent movement execution. 


\section{RESULTS}

To test our hypothesis that motor planning modulates the neural state of early auditory cortex, we performed two separate experiments that used delayed movement tasks. This allowed us to separate motor planning-related modulations from the later motor and somatosensory-related modulations that occur during movement execution. In effect, these delayed movement tasks allowed us to ask whether the motor command being prepared—but not yet executed—can be decoded from neural activity patterns in early auditory cortex. In the first of these experiments, in each trial we had participants first prepare, and then execute (after a jittered delay interval) either a left or right hand object lift-and-replace action, which were cued by two nonsense auditory commands ("Compty" or "Midwig"; see Fig 1). Importantly, halfway throughout each experimental run, participants were required to switch the auditory command-to-hand mapping (i.e., if "Compty" cued a left hand object lift-and-replace action in the first half of the experimental run, then "Compty" would cue a right hand object lift-and-replace action in the second half of the experimental run; see Fig 1B). Critically, this design allowed us to examine early auditory cortex activity during the planning of two distinct hand actions (left versus right hand movements), with invariance to the actual auditory commands (e.g., "Compty") used to instruct those hand actions. In the second of these experiments, in each trial we had participants first prepare, and then execute (after a fixed delay interval) either a right hand object lift-and-replace action or a target-directed eye movement (see Fig. 4). Unlike in Experiment 1, both of these movements were instructed via change in the colour of the central fixation light, thus allowing us to examine early auditory cortex activity during the planning of two distinct effector movements (hand versus eye) in the absence of any direct auditory input (i.e., no auditory commands). As such, any neural differences in the auditory cortex prior to movement in both of these experiments is likely to reflect top-down modulations related to motor, and not bottom-up sensory, processing. 
A
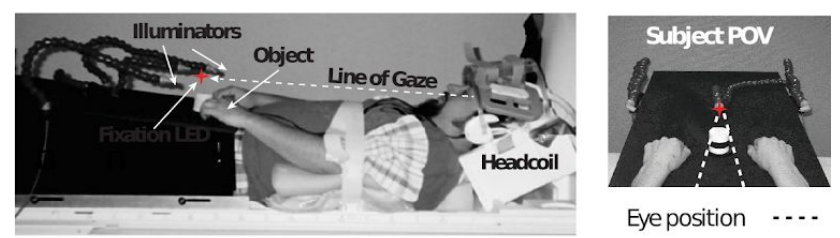

B
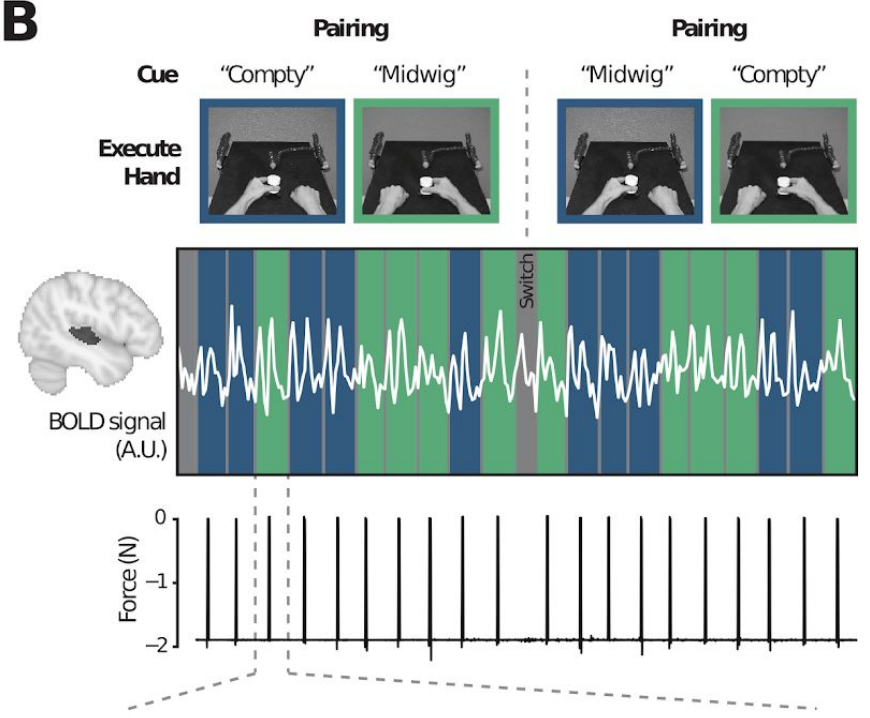

C

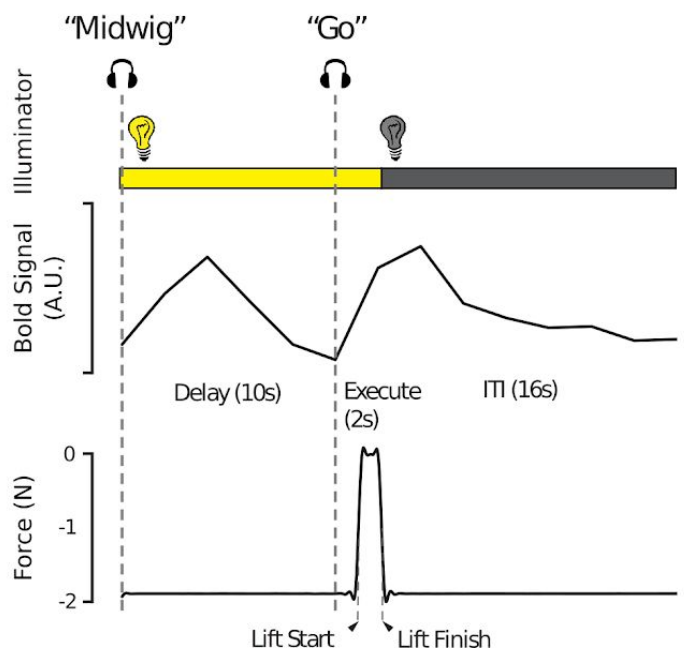

Figure 1. Experiment 1 setup and task overview.

A. MRI setup (left) and subject point-of-view (right) of the experimental workspace. Red star indicates the fixation LED placed above the object. Illuminator LEDs, attached to the flexible stalks, are shown on the left and right. B. Example fMRI run of 20 task trials. Color-coded columns (blue = left hand, green $=$ right hand) demarks each trial and the associated time-locked BOLD activity from superior temporal gyrus (STG; shaded in dark gray on cortex, left) of an exemplar subject is indicated by the overlaid white trace. Pairings between auditory cue ("Compty" or "Midwig") and hand (left or right) are indicated in the pictures above, and were reversed halfway through each run following a 'Switch' auditory cue, such that each hand is paired with each auditory cue in every experimental run (see Methods). The corresponding force sensor data, used to track object lifting, is shown below. C. Sequence of events and corresponding single-trial BOLD and force sensor data of an exemplar trial from a representative participant in which 'Midwig' cued a right-handed movement. Each trial begins with the hand workspace being illuminated while,

simultaneously, participants receive the auditory cue ("Compty" or "Midwig"). This is then followed by a jittered 6-12s Delay interval (10s in this exemplar trial). Next, an auditory "Go" cue initiates the start of the $2 \mathrm{~s}$ Execute epoch, during which the subject grasp-and-lifts the object (shown by the force trace; arrows indicate the start of the lift and object replacement). Following the $2 \mathrm{~s}$ Execute epoch, illumination of the workspace is extinguished and subjects then wait a fixed 16s intertrial interval (ITI) prior to onset of the next trial. See also Supplemental Fig. 1 for a more detailed overview of the trial sequence and the data obtained from a separate behavioural training session. 


\section{Experiment 1}

\section{Delay period decoding of hand information from early auditory cortex}

To determine whether signals related to hand movement planning influence early auditory cortex activity, we extracted the trial-related voxel patterns (beta coefficients) associated with the Delay (as well as Execute) epochs from early auditory cortex. To this end, we first functionally identified, using data from an independent auditory localizer task (see Methods), fMRI activity in the left and right superior temporal gyrus (STG). To provide greater specificity with regards to the localization of potential motor planning-related effects, we further delineated these STG clusters based on their intersections with Heschl's gyrus (HG) and the Planum Temporale (PT), two adjacent human brain regions associated with primary and higher-order cortical auditory processing, respectively ${ }^{16}$ (see Fig. $2 A, B$ for our basic approach). Next, for each of these 3 regions (STG, and its subdivisions into HG and PT) we used their z-scored Delay epoch voxel activity patterns as inputs to a support vector machine (SVM) binary classifier. In order to derive main-effects of hand information (i.e., examine decoding of upcoming left hand vs. right hand movements) versus auditory cue information (examine decoding of "Compty" vs. "Midwig" cues) and to increase the data used for classifier training, we performed separate analyses wherein we collapsed across auditory cue or hand trials, respectively. Our logic is that, when collapsing across auditory cue (i.e., re-labelling all trials based on the hand used), if we can observe decoding of hand information in auditory cortex during the Delay phase (prior to movement), then this information is represented with invariance to the cue, and thus sensory input (and vice versa).

Our analysis on the resulting classification accuracies (see Fig. 2D) revealed that information related to the upcoming hand actions to be performed (i.e., during the Delay epoch) was present in bilateral STG (left: $t_{14}=3.55, p=.002$; right: $t_{14}=2.34, p=.017$ ) and left $H G\left(t_{14}=2.43, p=.014\right)$. A significant effect was also found in right $H G$ but it did not survive FDR correction $\left(t_{14}=2.06, p=.029\right)$. Meanwhile, no significant decoding 
was found in left $\left(\mathrm{t}_{14}=-.074, \mathrm{p}=.529\right)$ or right $\left(\mathrm{t}_{14}=1.17, \mathrm{p}=.131\right) \mathrm{PT}$. By contrast, during the Execute epoch, we found that hand decoding was robust in all three areas in both hemispheres (all $\mathrm{p}<.001$, see Fig. 2D). Because our task did not pair the execution of hand movements to sound generation, and subjects would not have heard the auditory consequences associated with movement (e.g., object lifting and replacement) due to their wearing of headphones and the loud background noise of the scanner, these Execution results suggest that the modulation of auditory cortex activity is automatic and motor-related in nature ${ }^{13}$. Importantly, our finding of decoding in bilateral STG during the Delay period further suggests that this automatic and motor-related modulation also occurs well before execution, during movement preparation. An additional behavioural control experiment, performed prior to MRI testing (see Supplementary Material), suggests that the emergence of these hand-related effects are unlikely to be driven by systematic differences in eye position across trials ${ }^{17}$, since our trained participants exhibited highly stable fixations throughout the task (Supplemental Fig. 1).

In contrast to our motor-related hand decoding results, our analysis on the resulting classification accuracies for the sensory-related auditory cue ("Compty" vs. "Midwig") revealed that, during the Delay epoch, information related to the delivered verbal cue was present only in right STG $\left(t_{14}=3.71, p=.001\right.$, see Fig. 2D). Left PT also showed significant decoding $\left(\mathrm{t}_{14}=1.79, \mathrm{p}=.048\right)$, although this did not survive FDR correction. No cue decoding was found in the remaining ROls (all $p>.10$ ). Critically, consistent with the fact that this auditory cue information was delivered to participants only during the Delay epoch (i.e., participants always received a "Go" cue at the Execute epoch, regardless of trial identity), we also observed no evidence of cue decoding during the Execute epoch (all $p>.10)$. 
A

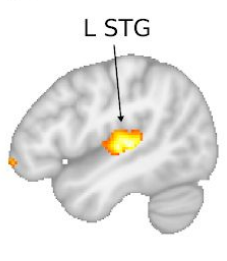

$x=-46$

B
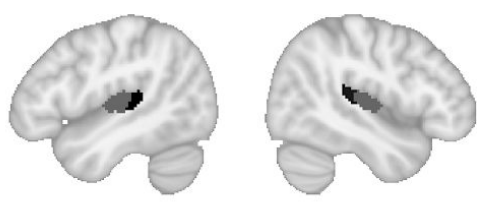

C

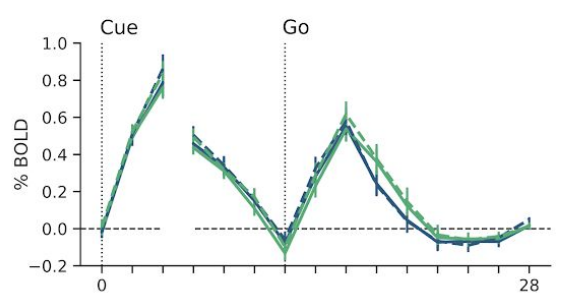

- Left Hand; "Compty"

- Right Hand; "Compty"

-- Left Hand; "Midwig"

-- Right Hand; "Midwig"

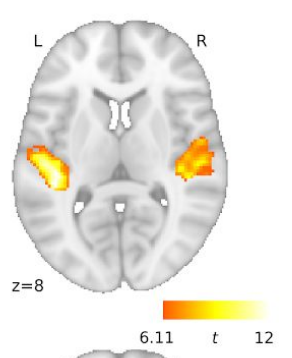

D
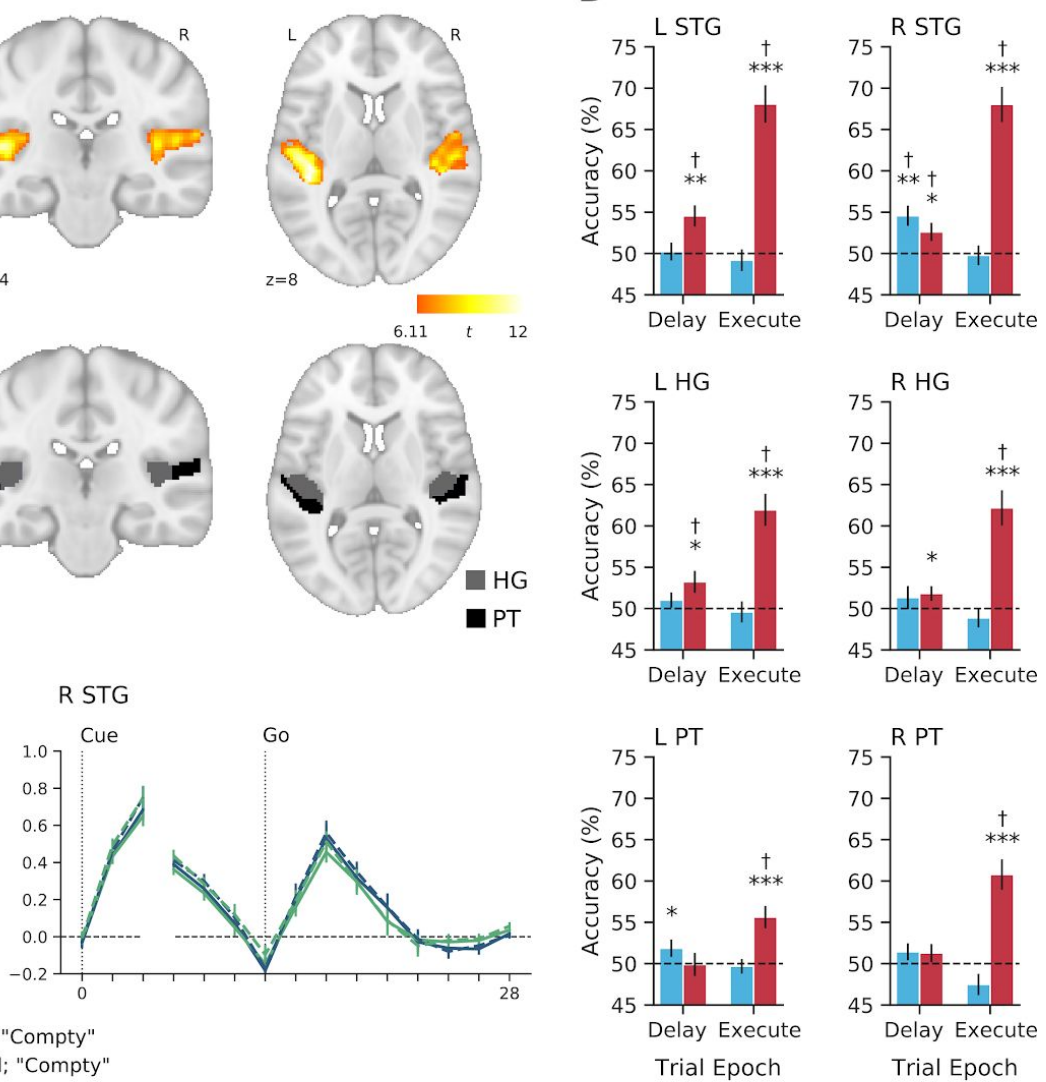

Hand: Left vs Right Cue: "Compty" vs "Midwig"

Figure 2. Decoding of hand information in early auditory cortex for Experiment 1. A. Left and right STG clusters defined at the group-level $(N=16)$ with the independent auditory localizer task using the contrast of Scrambled Speech $>$ Rest (see Methods). B. Delineation of Heschl's gyrus (HG) and Planum Temporale (PT), within each STG cluster from A. C. Group-averaged percent-signal change BOLD time courses for each trial type in left and right STG. The first three timepoints are time-locked to the onset of the Delay epoch and remaining time points are time-locked to onset of the Execute epoch. There is a high degree of overlap amongst the time courses for the different trial types. D. Decoding accuracies for hand (red) and cue (blue) information. Hand and cue decoding accuracies were analyzed separately in each epoch (Delay and Execute) using one-sample t-tests (one-tailed) against chance level (50\%). Error bars show \pm 1 SE of mean. ${ }^{*} p<.05,{ }^{* *} p<.01,{ }^{* *} p<.001, \dagger$ FDR corrected $q<.05$. 
Delay period decoding from auditory cortex mirrors that found in the motor system

To provide a basis for comparing and interpreting the above hand-related decoding effects in the auditory cortex, we also used the data from our experimental task to examine Delay epoch decoding in a positive control region, the dorsal premotor cortex (PMd). This region, which we independently identified PMd using a separate motor localizer task in our participants (see Methods), is well known to differentiate limb-related information during movement planning in both humans and nonhuman primates ${ }^{18,19}$. As shown in Supplemental Fig. 2C, we found a remarkably similar profile of limb-specific decoding in this motor-related region to that observed in the auditory cortex (area STG). In fact, direct comparisons between the magnitude of hand-related decoding in STG and PMd were non-significant (see Supplemental Fig. 2D). In summary, this PMd-result allows for two important observations. First, similar levels of action-related information can be decoded from early auditory cortex as from dorsal premotor cortex, the latter area known to have a well-established role in motor planning 20-22. Second, this Delay period decoding suggests that the representation of hand-related information evolves in a similar fashion prior to movement onset in both STG and PMd.

\section{Searchlight analyses reveal the representation of hand information in early auditory cortex prior to movement}

To complement our above ROI analyses, we also performed a group-level searchlight analysis within the wider auditory processing network, localized using our independent auditory localizer data (see Methods, Supplemental Fig. 3A). During the Delay epoch (see Fig. 3), two hand-related decoding clusters were identified in left STG, which includes a cluster centered on HG (212 voxels; peak, $x=-48, y=-24, z=10, t_{14}=8.31$, $\mathrm{p}<.00001$ ) and a cluster spanning anterolateral portions of left STG (321 voxels; peak, $x$ $\left.=-60, y=-2, z=-2, t_{14}=5.72, p<.000026\right)$. In the right hemisphere, one large cluster was revealed, which broadly spans across STG and superior temporal sulcus (519 voxels; peak, $\left.x=-50, y=-22, z=8, t_{14}=6.06, p<0.000015\right)$. Notably, when we 
examined cue-related decoding during the Delay epoch (i.e., decoding the auditory command 'Compty' vs. 'Midwig'), we found one cluster in right STG (272 voxels; peak, $x$ $\left.=58, y=-6, z=8, t_{14}=8.27, p<.00001\right)$, which did not overlap with the hand decoding clusters. This result suggests that, rather than a multiplexing of hand-related and cue-related within a common region of auditory cortex, separate subregions of the auditory system are modulated by motor-related (hand) versus sensory-related (auditory cue) information. In addition, the overlap of hand decoding clusters on bilateral HG and STG, as well as a cue decoding cluster in right STG, replicate our basic pattern of ROI-based results presented in Fig. 2.

A
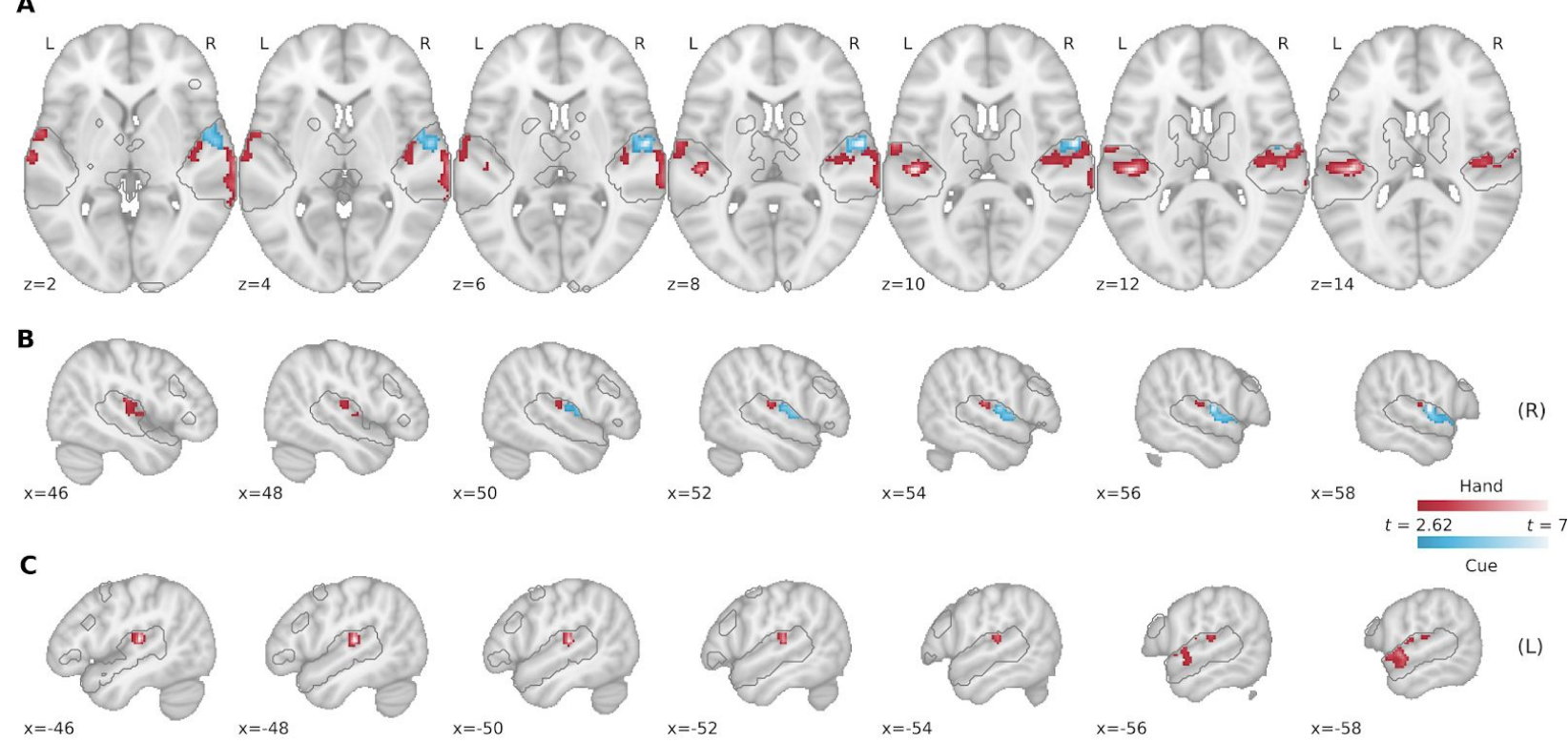

(L)

Figure 3. The representation of motor-related (hand) information during the Delay epoch in Experiment 1 is spatially distinct from the representation of sensory-related (auditory cue) information within superior temporal gyrus.

Searchlight analyses were restricted to a mask defined by significant voxels in a Intact Speech $>$ Rest contrast using an independent auditory localizer task (gray traced regions; see Methods). Group-level searchlight maps were thresholded at $t>2.62$ (one-tailed $p<.01$ ) and cluster-corrected at $p<.05$. A. Transverse slices of significant decoding clusters for hand (red) and auditory cue (blue) in early auditory areas during the Delay epoch. B. Sagittal slices of the right hemisphere. C. Sagittal slices of left hemisphere. 
A searchlight analysis using the Execute epoch data revealed a far more extensive pattern of hand decoding throughout the auditory network, with significant decoding extending all along the superior and middle temporal gyri bilaterally, and even into the basal ganglia and medial temporal cortex (see Supplemental Fig. 3B). By contrast, and in line with our ROI-based results, no cue decoding clusters were detected during the Execute epoch. These searchlight findings, when considered jointly with our ROI-based results, provide initial evidence that movement preparation selectively modulates neural activity patterns in early auditory cortex.

\section{Experiment 2}

Our analyses from Experiment 1 demonstrate that hand-related information (left vs. right hand) is represented bilaterally in early auditory cortex well prior to movement. What remains undetermined from this first study, however, is the extent to which these modulations in auditory cortex selectively reflect a motor-related process (i.e., a top-down modulation from the motor system). Indeed, one alternative explanation of the Experiment 1 results is that the decoding of limb-specific information could reflect some type of task-related association between auditory cues and actions, as has been recently suggested in the auditory cortex of nonhuman primates ${ }^{23}$. However, if auditory cortex is modulated in a top-down fashion via the motor system, then we would hypothesize - given the contralateral organization of the motor system ${ }^{24}$ and the existence of direct ipsilateral projections from motor to auditory cortex ${ }^{25}$ - that these modulations should be primarily contralateral in nature (i.e., the preparation of right hand movements should primarily modulate left auditory cortex and vice versa). The fact that both hands were used in Experiment 1 (and we observed decoding in bilateral auditory cortex) makes it difficult to unpack this hypothesis. Thus, to directly test this idea, in a second experiment we modified a classic task from primate neurophysiology used to dissociate motor- versus sensory-related coding during action planning ${ }^{26,27}$. In our version of this delayed movement task, in each trial, participants either grasped an object with their right hand or made an eye movement towards it (Fig. 4A). As such, if 
decoding in the auditory cortex is directly linked to the contralateral limb, then we would expect to only observe decoding in the left (and not right) auditory cortex. In addition, unlike in Experiment 1, wherein we used auditory commands to instruct actions at the onset of the Delay peoch, here we cued the two movements via a change in the colour of the fixation LED (Fig. 4A). As such, any Delay epoch decoding in the auditory cortex of the upcoming action to be performed (i.e., hand vs. eye movement) would be unrelated to any extraneous auditory inputs and can only be related to the motor effector to be used.

\section{Delay period decoding from early auditory cortex is contralateral in nature}

As in Experiment 1, a pattern classification analysis on Delay period voxel patterns revealed that information related to the upcoming effector to be used (hand vs. eye) could be decoded from the STG (see Fig. 4C). Critically, however, we found that this decoding during the Delay epoch was only observed in the contralateral (left), and not ipsilateral (right), STG (left: $t_{12}=3.58, p=.001$; right: $t_{12}=1.42, p=.091$ ). This is consistent with our hypothesis that the top-down, motor-related modulation of the auditory cortex, prior to movement, should occur in a contralateral fashion. As a departure from the effects observed in Experiment 1, however, we did not find that significant decoding was constrained solely to left $H G\left(t_{12}=1.32, p=.106\right.$; decoding in left $\mathrm{PT}$ and right $\mathrm{HG}$ and $\mathrm{PT}$ were all $\mathrm{p}>.10)$. During the Execute epoch, as in Experiment 1, we found that effector-related decoding was robust in all three areas and in both hemispheres (all $p<.008$ ). Together, these results support the key observation from Experiment 1 that the auditory cortex contains effector-specific information prior to movement onset. In addition, this second experiment further shows (1) that this effector-specific information is represented in the auditory cortex contralateral to the (right) hand being used, and (2) that this modulation occurs even in the absence of auditory inputs at the onset of the Delay epoch. Indeed, with respect to the latter point, it is noteworthy that decoding is observed in left STG, even when there is no net change 
in auditory activation prior to movement (see Fig 4B for baseline activation levels during the Delay epoch).

A

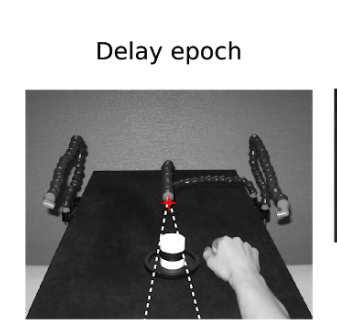

Execute epoch

Look

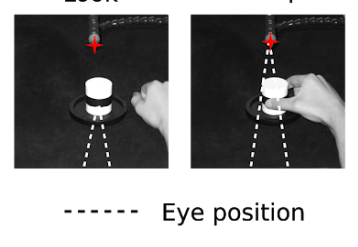

Color cue

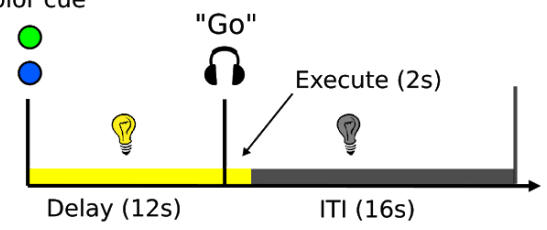

B

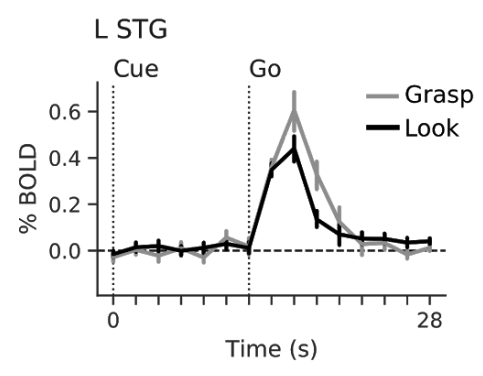

D
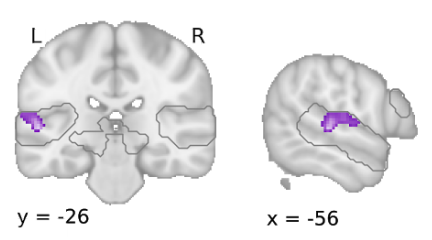

R STG

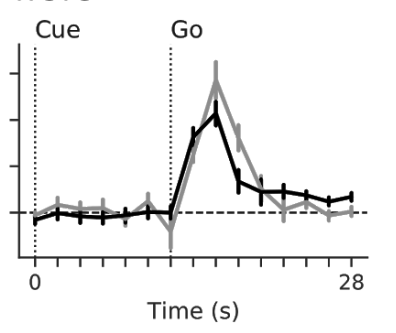

C
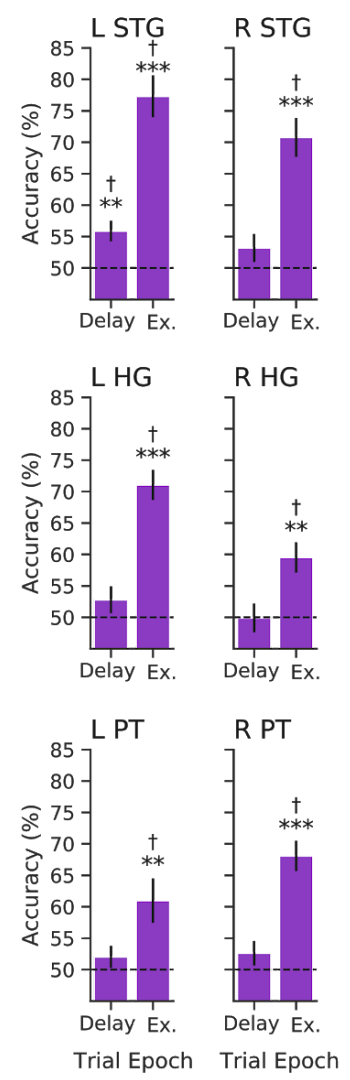

$\mathbf{E}$
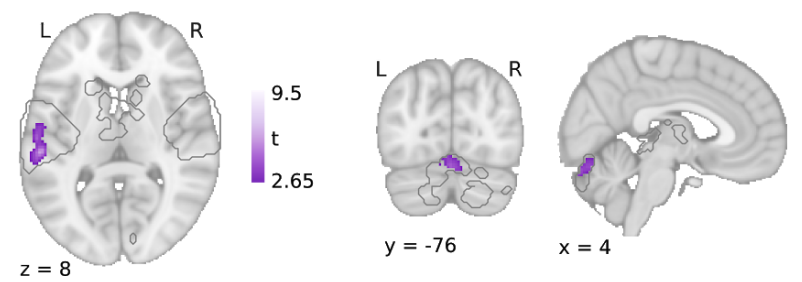

Figure 4. Experiment 2 shows that effector-specific decoding in auditory cortex is contralateral in nature. A. Subject point of view of the experimental workspace in Delay and Execute epochs (top) and trial flow (bottom). Red star indicates the fixation illuminator LED. B. Group-averaged BOLD timecourses for the Grasp (grey) and Eye movement ('Look', black) conditions in left and right STG. Note the absence of any net change in BOLD activity during the delay epoch for both trial types. C. Decoding accuracies for Grasp vs Look conditions during the Delay and Execute epochs in all auditory ROls. Decoding accuracies for each epoch were tested against chance decoding $(50 \%)$ using one-sample t-tests. Error bars show \pm 1 SE of mean. ${ }^{*} p<.05,{ }^{* *}$ $p<.01,{ }^{* * *} p<.001,+$ FDR corrected $q<.05$. D. Purple clusters show searchlight 
results of significant decoding for hand vs. eye information in contralateral (left) auditory cortex during the Delay epoch. As in Experiment 1, searchlight analyses were restricted to a mask defined by significant voxels in a Intact Speech > Rest contrast using the independent auditory localizer task data (gray traced regions; see Methods). Group-level searchlight maps were thresholded at $t>2.65$ (one-tailed $p<.01$ ) and cluster-corrected at $p<.05$. E. Significant decoding in the cerebellum, for the same analysis as in D.

As in Experiment 1, it is useful to interpret these above decoding effects in left STG with respect to a positive control region, like PMd, known to distinguish upcoming hand versus eye movements during the Delay epoch in humans ${ }^{28}$. As shown in the Supplementary Fig. 4, we find a similar level of decoding of effector-specific information in left PMd $\left(t_{12}=2.78, p=.008\right)$ as that observed in left STG above. This shows that motor-related information is just as decodable from the auditory cortex as it is from a motor-related region, like PMd.

\section{Searchlight analyses reveal the representation of effector-specific information in contralateral auditory cortex prior to movement}

To complement our ROI analyses and bolster our observations in Experiment 1, we again performed a group-level searchlight analysis within the wider auditory processing network (denoted by the gray traces in Fig. 4D,E). During the Delay epoch (see Fig. 4D), we identified a large effector-specific (hand vs. eye) decoding cluster in left STG (308 voxels; peak, $x=-54, y=-28, z=4, t_{13}=9.49, p<.00001$ ), as well as a smaller cluster in cerebellum (150 voxels; peak, $x=6, y=-78, z=-18, t_{13}=4.47, p<.00026$, see Fig. 4E). A searchlight analysis using the Execute epoch data replicated our general observations from Experiment 1 that movement execution elicits far more widespread activity through the wider auditory network (see Supplemental Fig 5). These searchlight results, when considered jointly with our ROI-based findings, provide strong supporting evidence that movement preparation primarily modulates neural activity in the contralateral auditory cortex. 


\section{DISCUSSION}

Here we have shown, using $\mathrm{fMRI}$ and two separate experiments involving delayed movement tasks, that effector-specific information (i.e., left vs. right hand in Experiment 1 , and hand vs. eye in Experiment 2) can be decoded from pre-movement neural activity patterns in early auditory cortex. In Experiment 1, we showed that effector-specific decoding was invariant to the auditory cue used to instruct the participant on which hand to use. Separately, with our searchlight analyses, we also found that the decoding of this hand-related information occured in a separate subregion of auditory cortex than the decoding of the auditory cue that instructed the motor action. In Experiment 2, we showed that effector-specific decoding in the auditory cortex is in fact contralateral to the hand being used, and occurs even in the absence of any auditory input during the delay period (i.e., when movements are instructed via a visual cue). Taken together, these findings suggest that a component of action planning, beyond preparing motor areas for the forthcoming movement, involves modulating early sensory cortical regions. Such modulation may enable these areas to more effectively participate in the filtering and processing of task-specific sensory signals that arise during the unfolding movement itself.

With respect to motor-related brain areas (e.g., the primary, premotor, and supplementary motor cortices), several hypotheses have been proposed about the role of planning-related activity. Some researchers have suggested that planning activity codes a variety of different movement parameters (e.g., direction, speed), with a view that it represents some subthreshold version of the forthcoming movement ${ }^{1,29-35}$. More recent work, examining populations of neurons in motor areas, has instead suggested that movement planning involves setting up the initial state of the population, such that movement execution can unfold naturally through transitory neural dynamics ${ }^{3,21,36-39}$. With respect to this newer framework, our results suggest that motor planning may also involve setting up the initial state of primary sensory cortical areas. Whereas the neural activity patterns that unfold during movement execution in motor areas are thought to 
regulate the timing and nature of descending motor commands ${ }^{3,40}$, such activity in primary sensory areas could instead regulate the timing and nature of the filtering of incoming sensory signals.

One idea is that motor preparation signals originating from the motor system could tune early sensory areas for a role in sensory prediction. Prediction of the sensory consequences of action is essential for the accurate sensorimotor control of movement, per se, and also provides a mechanism for distinguishing between self-generated and externally generated sensory information ${ }^{4}$. The critical role of prediction in sensorimotor control has been well documented in the context of object manipulation tasks ${ }^{5,6}$, like those used in our experiments. The control of such tasks centers around contact events, which give rise to discrete sensory signals in multiple modalities (e.g., auditory, tactile) and represent subgoals of the overall task. Thus, in the grasp, lift, and replace task that our participants performed in both Experiments 1 and 2, the brain automatically predicts the timing and nature of discrete sensory signals associated with contact between the digits and object, as well as the breaking, and subsequent making, of contact between the object and surface; events that signify successful object grasp, lift-off, and replacement, respectively. By comparing predicted and actual sensory signals associated with these events, the brain can monitor task progression and launch rapid corrective actions if mismatches occur ${ }^{41}$. These corrective actions are themselves quite sophisticated and depend on both the phase of the task and the nature of the mismatch ${ }^{5}$. Thus, outside the motor system, the preparation of manipulation tasks clearly involves forming what could be referred to as a 'sensory plan'; i.e., a series of sensory signals-linked to contact events-that, during subsequent movement execution, can be predicted based on knowledge of object properties and information related to outgoing motor commands (Johansson and Flanagan, 2009). In the context of the experimental tasks and results presented here, it would make sense that such 'sensory plans' be directly linked to the acting limb (and thus, decodable at the level of 
auditory cortex), given that successful sensory prediction, and rapid motor correction, would necessarily require direct knowledge of the hand being used.

The disambiguation of self- and externally generated sensory information is thought to rely on cancelling, or attenuating, the predictable sensory consequences of movements 4,42. Such sensory predictions for use in sensory cancellation are generally thought to be represented in primary sensory areas. According to this view, an efference copy of descending motor commands, associated with movement execution, is transmitted in a top-down fashion to early sensory cortices in order to attenuate self-generated sensory information ${ }^{43,44}$. By contrast, sensory predictions for use in upcoming sensorimotor control, are thought to be represented in the same frontoparietal circuits involved in movement planning and execution 45,46. According to this view, incoming sensory information, associated with movement execution, is transmitted in a bottom-up fashion from early sensory areas to frontoparietal circuits for mismatch detection and movement correction ${ }^{47-49}$. Although here we observe our task-related modulations in early sensory (auditory) cortex, we think it is unlikely that it purely reflects a sensory cancellation process. First, sensory attenuation responses in primary auditory cortex have been shown to occur about two hundred milliseconds prior to movement onset ${ }^{13,14}$, whereas the effector-specific modulations of auditory cortex we report here occurred, at minimum, several seconds prior to movement onset (as revealed through our analyses linked to the onset of the Delay epoch). Second, any sensory attenuation would be expected to occur in bilateral auditory cortex, given that the sound generated from movement would be necessarily processed bi-aurally, which contrasts with our effector-related decoding in only the contralateral auditory cortex (in Experiment 2). Given these considerations, a more likely explanation of our results is that they reflect the motor system preparing the state of auditory cortex, in a top-down fashion, to process future auditory signals for a role in forthcoming sensorimotor control. 
There are three lines of support for the notion that the effector-specific decoding observed here in auditory cortex is the result of a top-down, motor-related modulation. First, in the MRI scanner environment (i.e., loud background noise, subjects' wearing of headphones), our participants could not have heard the auditory consequences of their actions; e.g., sounds associated with contacting, lifting, and replacing the object. This argues that our reported modulation of auditory cortex is automatic in nature, and not linked to any sensory reafference or attentional processes see 50,51. Second, our searchlight analyses in Experiment 1 identified separate clusters in STG that decoded hand information (in early auditory cortex) from those that decoded auditory cue information (in higher-order auditory cortex). The fact that different subregions of auditory cortex are modulated prior to movement for motor (i.e., discriminating left vs. right hand) versus auditory language information (i.e., discriminating 'Compty' vs. 'Midwig') is consistent with the notion that our hand-related decoding effects are invariant to sensory input. Finally, and perhaps most compellingly, in Experiment 2 the decoding of effector-specific information was found to be contralateral to the hand being used in the task. Such lateralization is a main organizational feature of movement planning and control throughout the motor system ${ }^{24}$, and is consistent with neuroanatomical tracing work showing that the motor cortex has direct projections to the auditory cortex within the same hemisphere ${ }^{25}$. By contrast, no such organization exists in the cortical auditory system, with lateralization instead thought to occur along different dimensions (e.g., language processing ${ }^{9}$ ). Critically, the contralaterality observed in our Experiment 2 also rules out the possibility that our results are merely the byproduct of an auditory working-memory/rehearsal process, whereby, during the delay period, participants translate an auditory (e.g., 'Compty' in Experiment 1) or visual cue (e.g., change in fixation LED colour in Experiment 2) into the corresponding auditory instruction (e.g., subvocalize to themselves "Right hand"). Indeed, prior fMRI work has shown that the maintenance of auditory information in working memory recruits bilateral auditory cortex ${ }^{10}$. Here, however, we find decoding of motor effector information in bilateral auditory cortex when both hands are interchangeably used in the task 
(Experiment 1) and find only contralateral decoding of motor effector information when only one of the hands is used in the task (Experiment 2). These observations, when taken together, support the notion that the signals being decoded from the auditory cortex prior to movement have a motor, rather than sensory, origin.

Prior work has also demonstrated that tactile input alone is capable of driving auditory cortex activity ${ }^{52-55}$, indicating a potential role for auditory cortex in multisensory integration. As noted above, the control of object manipulation tasks involves accurately predicting discrete sensory events that arise in multiple modalities, including tactile and auditory ${ }^{6}$. It is plausible then that some of the pre-movement auditory cortex modulation described here reflects the predicted tactile events arising from our task (e.g., object contact, lift-off and replacement), which we would also expect to be linked to the acting hand (and thus, decodable). Though we cannot disentangle this possibility in the current study, it does not undercut our main observation that early auditory cortex is selectively modulated as a function of the movement being prepared; nor does it undercut our interpretation-that such modulation is likely linked to sensorimotor prediction.

In summary, here we show that, prior to movement, neural activity patterns in early auditory cortex carry information about the movement effector to be used in the upcoming action. This result supports the hypothesis that sensorimotor planning, which is critical in preparing neural states ahead of movement execution ${ }^{21}$, not only occurs in motor areas but also in primary sensory areas. While further work is required to establish the precise role of this movement-related modulation, our findings add to a growing line of evidence indicating that early sensory systems are directly modulated by sensorimotor computations performed in higher-order cortex ${ }^{56-59}$ and not merely low-level relayers of incoming sensory information ${ }^{23,60-62}$. 


\section{MATERIALS \& METHODS}

\section{Participants}

Sixteen healthy right-handed subjects (8 females, 21-25 years of age) participated in Experiment 1, which involved one behavioural testing session followed by two fMRI testing sessions (a localizer testing session, and then the experimental testing session, performed on separate days approximately 1-2 weeks apart). Fifteen healthy right-handed subjects (8 females, 20-32 years of age) participated in Experiment 2, which involved one behavioural testing session and one fMRI testing session. Right-handedness was assessed with the Edinburgh handedness questionnaire ${ }^{63}$. Informed consent and consent to publish were obtained in accordance with ethical standards set out by the Declaration of Helsinki (1964) and with procedures cleared by the Queen's University Health Sciences Research Ethics Board. Subjects were naïve with respect to the hypotheses under evaluation and received monetary compensation for their involvement in the study. Data from one subject in Experiment 1 and from two subjects in Experiment 2 were excluded from further analyses due to data collection problems in the experimental testing sessions, resulting in final sample sizes of 15 and 13 subjects, respectively. Meanwhile, all 16 subjects from Experiment 1 were used for the localizer testing session.

\section{Experiment 1}

\section{Experimental apparatus}

The experimental setup for both the localizer and experimental testing sessions consisted of a black platform placed over the waist and tilted away from the horizontal at an angle $\left(\sim 15^{\circ}\right)$ to maximize comfort and target visibility. The MRI head coil was tilted slightly $\left(\sim 20^{\circ}\right)$ and foam cushions were used to give an approximate overall head tilt of $30^{\circ}$. To minimize limb-related artifacts, subjects had the right and left upper-arms braced, limiting movement of the arms to the elbow and thus creating an arc of reachability for each hand. The exact placement of object stimuli on the platform was adjusted to match each subject's arm length such that all required actions were 
comfortable and ensured that only movement of the forearm, wrist and fingers was required. The platform was illuminated by two bright white Light Emitting Diodes (LEDs) attached to flexible plastic stalks (Loc-Line, Lockwood Products, Lake Oswego, OR, USA) located to the left and right of the platform. To control for eye movements, a small red fixation LED, attached to a flexible plastic stalk, was positioned above the hand workspace and located $\sim 5 \mathrm{~cm}$ beyond the target object position (such that the object appeared in the subject's lower visual field). Experimental timing and lighting were controlled with in-house software created with $\mathrm{C}++$ and MATLAB (The Mathworks, Natnick, MA). Throughout fMRI testing, the subject's hand movements were monitored using an MR-compatible infrared-sensitive camera (MRC Systems GmbH, Heidelberg, Germany), optimally positioned on one side of the platform and facing towards the subject. The videos captured during the experiment were analyzed offline to verify that the subjects were performing the task as instructed and to identify error trials.

\section{Auditory Localizer Task}

A separate, block-design localizer task was collected to independently identify auditory cortex and higher-order language regions of interest (ROIs) for use in the analyses of main experimental task. This auditory localizer task included three conditions: (1) Intact speech trials (Intact), which played one of 8 unique excerpts of different speeches; (2) scrambled speech trials (Scrambled), which were incoherent signal-correlated noise versions of the speech excerpts (i.e. applying an amplitude envelope of the speech to uniform Gaussian white noise, ensuring that the noise level was utterance-specific and exactly intense enough at every moment to mask the energy of the spoken words); and (3) rest trials (Rest), in which no audio was played (subjects thus only heard background MRI scanner noise during this time). Trials lasted $20 \mathrm{~s}$ each and alternated, in pseudo-random order, between Intact Speech, Scrambled Speech, and Rest for a total of 24 trials in each experimental run. In addition, a $20 \mathrm{~s}$ baseline block was placed at the beginning of each experimental run. Each localizer run totaled $500 \mathrm{~s}$ and participants completed 2 of these runs during testing (resulting in 16 repetitions per 
experimental condition per subject). To encourage that participants maintained attention throughout this auditory localizer run, they were required to monitor each of the Intact speeches and let the experimenter know, following the run, whether any of them were repeated. This repeat happened in only one of the experimental runs and each and every subject correctly identified the repeat and non-repeat run (100\% accuracy).

\section{Motor Localizer task}

Four experimental runs of a motor localizer task were also collected alongside the auditory localizer task, which constituted a block-design protocol that alternated between subtasks designed to localize eight separate motor functions. Task set up and details for all eight conditions are described in Supplemental Fig. 2. The hand grasping condition from this localizer task was used to define dorsal premotor cortex (PMd), which we used as a basis for comparison with our auditory cortex decoding findings (see Results).

The motor and auditory localizer testing session lasted approximately 2 hours and included set-up time, one 7.5 minute high-resolution anatomical scan and 6 functional scanning runs, wherein subjects alternated between performing two runs of the motor localizer task and one run of the auditory localizer, twice. A brief ( 10 minute) practice session was carried out before the localizer testing session in the MRI control room in order to familiarize participants with localizer tasks.

\section{Main Experimental Task}

In our experimental task (see Fig. 1), we used a delayed movement paradigm wherein, on each individual trial, subjects were first auditorily cued (via headphones) to prepare either a left vs. right hand object grasp-and-lift action on a centrally located cylindrical object (1.9 N weight). Then, following a variable delay period, they were prompted to execute the prepared hand action. At the start of each event-related trial (see Fig. 1C), simultaneously with the LED illuminator lights going on (and the subject's workspace 
being illuminated), subjects received one of two nonsense speech cues, "Compty" or "Midwig". For a given trial, each nonsense speech cue was paired with a corresponding hand action (e.g., subjects were instructed that, for a predetermined set of trials, "Compty" cued a left hand movement whereas "Midwig" cued a right hand movement). [Note that nonsense speech commands were chosen because semantically meaningful words such as "left" and "right" would already have strong cognitive associations for participants.] Following the delivery of the auditory command, there was a jittered delay interval of 6-12 s (a Gaussian random jitter with a mean of $9 \mathrm{~s}$ ), after which the verbal auditory command "Go" was delivered, prompting subjects to execute the prepared grasp-and-lift action. For the execution phase of the trial, subjects were required to precision grasp-and-then-lift the object with their thumb and index finger $(\sim 2 \mathrm{~cm}$ above the platform, via a rotation of the wrist), hold it in midair for $\sim 1 \mathrm{~s}$, and then replace it. Subjects were instructed to keep the timing of each hand action as similar as possible throughout the study. Two seconds following the onset of this "Go" cue, the illuminator lights were extinguished, and subjects then waited $16 \mathrm{~s}$ for the next trial to begin (intertrial interval, ITI). Throughout the entire time course of the trial, subjects were required to maintain gaze on the fixation LED.

These event-related trials were completed in two separate blocks per experimental run. At the beginning of each experimental run, the experimenter informed subjects of the auditory-hand mapping to be used for the first 10 event-related trials of the experimental run (e.g. "Compty" for left hand (left hand) movements, "Midwig" for right hand (right hand) movements; 5 intermixed trials of each type). After the 10th trial, the illuminator was turned on (for a duration of $6 \mathrm{~s}$ ) and subjects simultaneously heard the auditory command "Switch" (following by a $16 \mathrm{~s}$ delay). This indicated that the auditory-hand mapping would now be reversed for the remaining 10 event-related trials (i.e., "Compty" would now cue a right hand movement whereas "Midwig" would now cue a left hand movement). The sequential ordering of this auditory-hand mapping was counterbalanced across runs, and resulted in a total of 4 different auditory-hand 
mappings (and thus, trial types) per experimental run: Compty-left hand, Compty-right hand, Midwig-left hand, and Midwig-right hand (with 5 repetitions each; 20 trials in total per run). With the exception of the blocked nature of these trials, these trial types were pseudorandomized within a run and counterbalanced across all runs so that each trial type was preceded and followed equally often by every other trial type across the entire experiment.

Separate practice sessions were carried out before the actual fMRI experiment to familiarize subjects with the delayed timing of the task. One of these sessions was conducted before subjects entered the scanner (See Behavioural Control Experiment below) and another was conducted during the anatomical scan (collected at the beginning of the experimental testing session). The experimental testing session for each subject lasted approximately 2 hours and included set-up time, one 7.5 minute high-resolution anatomical scan (during which subjects could practice the task) and eight functional scanning runs (for a total of 160 trials; 40 trials for each auditory-motor mapping). Each functional run (an example run shown in Fig. 1B) had a duration of 576 $\mathrm{s}$, with a 30-60 s break in between each run. Lastly, a resting state functional scan, in which subjects lay still (with no task) and only maintained gaze on the fixation LED, was performed for 12 minutes (data not analyzed here).

During MRI testing, we also tracked subjects' behaviour using an MRI-compatible force sensor located beneath the object (Nano 17 F/T sensors; ATI Industrial Automation, Garner, NC), and attached to our MRI platform. This force sensor, which was capped with a flat circular disk (diameter of $7.5 \mathrm{~cm}$ ), supported the object. The force sensor measured the vertical forces exerted by the object (signals sampled at $500 \mathrm{~Hz}$ and low-pass filtered using a $5^{\text {th }}$ order, zero-phase lag Butterworth filter with a cutoff frequency of $5 \mathrm{~Hz}$ ), allowing us to track both subject reaction time (RT), which we define as the time from the onset of the "Go" cue to object contact (Mean $=1601 \mathrm{~ms}, \mathrm{SD}=$ $389 \mathrm{~ms}$ ), and movement time (MT), which we define as the time from object lift to 
replacement $($ Mean $=2582 \mathrm{~ms}, \mathrm{SD}=662 \mathrm{~ms})$, as well as generally monitor task performance. Note that we did not conduct eye tracking during this or any of the other MRI scan sessions because of the difficulties in monitoring gaze in the head-tilted configuration with standard MRI-compatible eye trackers (due to occlusion from the eyelids) ${ }^{56,64,65}$.

\section{Experiment 2}

This study and experimental setup was similar to Experiment 1, with the exception that: (1) participants performed either a right hand object grasp-and-lift action on a centrally located cylindrical object (1.9 N weight) or a target-directed eye movement towards that same object (i.e., two experimental conditions), (2) the Delay epoch was a fixed duration (12 s), and (3) subjects were cued about the upcoming movement to be executed via a $0.5 \mathrm{~s}$ change in the fixation LED colour (from red to either blue or green, with the colour-action mapping being counterbalanced across subjects; i.e., a LED change to blue cued a grasp action in half the subjects, and cued an eye movement in the other half of subjects). The eye movement action involved the subject making a saccadic eye movement from the fixation LED to the target object, holding that position until the illuminator LEDs were extinguished, and then returning their gaze to the fixation LED. The two trial types, with 5 repetitions per condition (10 trials total), were pseudorandomized as in Experiment 1. Each subject participated in at least eight functional runs.

\section{Data Acquisition and Analysis}

Subjects were scanned using a 3-Tesla Siemens TIM MAGNETOM Trio MRI scanner located at the Centre for Neuroscience Studies, Queen's University (Kingston, Ontario, Canada). An identical imaging protocol was used for both Experiments 1 and 2, with the exception of slice thickness (Experiment $1=4 \mathrm{~mm}$; Experiment $2=3 \mathrm{~mm}$ ). In both experiments, MRI volumes were acquired using a T2*-weighted single-shot gradient-echo echo-planar imaging acquisition sequence (time to repetition $=2000 \mathrm{~ms}$, 
in-plane resolution $=3 \mathrm{~mm} \times 3 \mathrm{~mm}$, time to echo $=30 \mathrm{~ms}$, field of view $=240 \mathrm{~mm} \times 240$ $\mathrm{mm}$, matrix size $=80 \times 80$, flip angle $=90^{\circ}$, and acceleration factor (integrated parallel acquisition technologies, iPAT) $=2$ with generalized auto-calibrating partially parallel acquisitions reconstruction). Each volume comprised 35 contiguous (no gap) oblique slices acquired at a $\sim 30^{\circ}$ caudal tilt with respect to the plane of the anterior and posterior commissure (AC-PC). Subjects were scanned in a head-tilted configuration, allowing direct viewing of the hand workspace. We used a combination of imaging coils to achieve a good signal to noise ratio and to enable direct object workspace viewing without mirrors or occlusion. Specifically, we tilted $\left(\sim 20^{\circ}\right.$ degrees) the posterior half of the 12-channel receive-only head coil (6-channels) and suspended a 4-channel receive-only flex coil over the anterior-superior part of the head. An identical T1-weighted ADNI MPRAGE anatomical scan was also collected for both Experiments 1 and 2 (time to repetition $=1760 \mathrm{~ms}$, time to echo $=2.98 \mathrm{~ms}$, field of view $=192 \mathrm{~mm} \mathrm{x}$ $240 \mathrm{~mm} \times 256 \mathrm{~mm}$, matrix size $=192 \times 240 \times 256$, flip angle $=9^{\circ}, 1 \mathrm{~mm}$ isotropic voxels).

\section{fMRI data preprocessing}

Preprocessing of functional data collected in the localizer testing session, and Experiments 1 and 2, was performed using fMRIPrep 1.4.1 ${ }^{66}$, which is based on Nipype $1.2 .0^{67,68}$.

\section{Anatomical data preprocessing}

The T1-weighted (T1w) image was corrected for intensity non-uniformity (INU) with N4BiasFieldCorrection ${ }^{69}$, distributed with ANTs 2.2.0 ${ }^{70}$, and used as T1W-reference throughout the workflow. The T1w-reference was then skull-stripped with a Nipype implementation of the antsBrainExtraction.sh workflow (from ANTs), using OASIS30ANTs as target template. Brain tissue segmentation of cerebrospinal fluid (CSF), white-matter (WM) and gray-matter (GM) was performed on the brain-extracted T1w using fast (FSL 5.0.9, ${ }^{71}$ ). Brain surfaces were reconstructed using recon-all (FreeSurfer $6.0 .1,{ }^{72}$ ), and the brain mask estimated previously was refined with a 
custom variation of the method to reconcile ANTs-derived and FreeSurfer-derived segmentations of the cortical gray-matter of Mindboggle ${ }^{73}$. Volume-based spatial normalization to standard space (voxel size $=2 \times 2 \times 2 \mathrm{~mm}$ ) was performed through nonlinear registration with antsRegistration (ANTs 2.2.0), using brain-extracted versions of both T1w reference and the T1w template. The following template was selected for spatial normalization: FSL's MNI ICBM 152 non-linear 6th Generation Asymmetric Average Brain Stereotaxic Registration Model [4; TemplateFlow ID: MNI152NLin6Asym].

\section{Functional data preprocessing}

For each BOLD run per subject (across all tasks and/or sessions), the following preprocessing was performed. First, a reference volume and its skull-stripped version were generated using a custom methodology of fMRIPrep. The BOLD reference was then co-registered to the T1w reference using bbregister (FreeSurfer) which implements boundary-based registration ${ }^{75}$. Co-registration was configured with nine degrees of freedom to account for distortions remaining in the BOLD reference. Head-motion parameters with respect to the BOLD reference (transformation matrices, and six corresponding rotation and translation parameters) are estimated before any spatiotemporal filtering using mcflirt (FSL 5.0.9, ${ }^{76}$ ). BOLD runs were slice-time corrected using 3dTshift from AFNI $20160207{ }^{77}$. The BOLD time-series were normalized by resampling into standard space. All resamplings were performed with a single interpolation step by composing all the pertinent transformations (i.e. head-motion transform matrices, and co-registrations to anatomical and output spaces). Gridded (volumetric) resamplings were performed using antsApplyTransforms (ANTs), configured with Lanczos interpolation to minimize the smoothing effects of other kernels 
Many internal operations of fMRIPrep use Nilearn $0.5 .2^{79}$, mostly within the functional processing workflow. For more details of the pipeline, see the section corresponding to workflows in fMRIPrep's documentation.

\section{Post-processing}

Additional post-processing was performed for specific analyses. Normalized functional scans were temporally filtered using a high-pass filter (cutoff $=0.01 \mathrm{~Hz}$ ) to remove low-frequency noise (e.g. linear scanner drift), either as part of GLMs (see below) or directly (as in time-point decoding analyses). For the localizer data, normalized functional scans were spatially smoothed (6mm FWHM Gaussian kernel; SPM12) prior to GLM estimation to facilitate subject overlap. [Note that no spatial smoothing was performed on the experimental task data sets, wherein multi-voxel pattern analyses were performed.]

\section{Error trials}

Error trials were identified offline from the videos recorded during the experimental testing session and were excluded from analysis by assigning these trials predictors of no interest. Error trials included those in which the subject performed the incorrect instruction (Experiment 1: 9 trials, 4 subjects; Experiment 2: 1 trial, 1 subject) or contaminated the delay phase data by slightly moving their limb or moving too early (Experiment 1: 7 trials, 4 subjects; Experiment 2: 1 trial, 1 subject). The fact that subjects made so few errors when considering the potentially challenging nature of the tasks (e.g., in Experiment 1 having to remember whether "Compty" cued a left hand or right hand movement on the current trial) speaks to the fact that subjects were fully engaged during experimental testing and very well practiced at the task prior to participating in the experiment. 


\section{Statistical Analyses}

\section{General Linear Models}

For the localizer task analyses, we carried out subject-level analysis using SPM12's first-level analysis toolbox to create general linear models (GLM) for each task (auditory and motor). Each GLM featured condition predictors created from boxcar functions convolved with a double-gamma hemodynamic response function (HRF), which were aligned to the onset of each action/stimulus block with durations dependent on block length (i.e., 10 imaging volumes for both localizer tasks). Temporal derivatives of each predictor and subjects' six motion parameters obtained from motion correction were included as additional regressors. The Baseline/Fixation epochs were excluded from the model; therefore all regression coefficients (betas) were defined relative to the baseline activity during these time points.

In the experimental tasks, we employed a Least-Squares Separate procedure (Mumford et al., 2012), with separate GLM models for each individual trial's Delay and Execute epochs were generated in order to extract beta coefficient estimates for decoding analyses (e.g., In Experiment 1: 20 trials $\times 2$ epochs $\times 8$ runs $=320$ GLMs). The regressor of interest in each model consisted of a boxcar regressor aligned to the start of the epoch, with a duration corresponding to the length of the cue. In both Experiments, all cues were modeled with 0.5 s durations: the auditory command cue ('Compty' or 'Midwig') and the visual cue (fixation LED colour change) at the start of the Delay epoch in Experiment 1 and 2, respectively; and the auditory 'Go' cue at the start of the Execute epoch in both experiments. For each GLM, we included a second regressor that comprised of all remaining trial epochs in the experimental run. Each regressor was then convolved with a double-gamma HRF, and temporal derivatives of both regressors were included along with subjects' six motion parameters obtained from motion correction. Isolating the regressor of interest in this single-trial fashion reduces 
regressor collinearity, and has been shown to be advantageous in estimating single-trial voxel patterns and for multi-voxel pattern classification ${ }^{80}$.

\section{Region of interest (ROI) selection}

Regions of interests (ROI) were identified based on second-level (group) analyses of first-level contrast images from each subject. Early auditory cortex ROls were identified for both Experiment 1 and 2 by thresholding a Scrambled Speech > Rest group contrast at an uncorrected voxelwise threshold of $p<10^{-5}$. This procedure identified tight superior temporal gyrus (STG) activation clusters in left and right Heschl's gyrus (HG), the anatomical landmark for primary (core) auditory cortex ${ }^{81-84}$, and more posteriorly on the superior temporal plane (Planum Temporale, PT). We verified these locations by intersecting region masks for HG and PT obtained from the Harvard-Oxford anatomical atlas ${ }^{85}$ with the masks of left and right STG clusters. This allowed us to define, for each participant, voxels that were active for sound that fell in anatomically defined HG and PT. We considered HG and PT separately since they are at different stages of auditory processing: HG is the location of the core, whereas the PT consists of belt and probably parabelt regions, as well as possibly other types of cortical tissue ${ }^{86}$. Since our PT activity is just posterior to HG, we suspect that this is probably in belt or parabelt cortex, one or two stages of processing removed from core. Lastly, a more expansive auditory and speech processing network was obtained using a Intact Speech > Rest contrast with an uncorrected height threshold of $p<.001$ and cluster-extent correction threshold of $p<.05$. Together, these were used as three-dimensional binary masks to constrain our analyses and interpretations of motor-related effects in the auditory system.

\section{Multi-voxel Pattern Analysis (MVPA)}

For the experimental task, MVPA was performed with in-house software using Python 3.7.1 with Nilearn v0.6.0 and Scikit-Learn v0.20.1 ${ }^{79}$. All analyses implement support vector machine (SVM) binary classifiers (libSVM) using a radial-basis function kernel and with fixed regulation parameter $(C=1)$ and gamma parameter (automatically set to 
the reciprocal of the number of voxels) in order to compute a hyperplane that best separated the trial responses. The pattern of voxel beta coefficients from the single-trial GLMs, which provided voxel patterns for each trial's Delay and Execute epochs, were used as inputs into the binary classifiers. These values were standardized across voxels such that each voxel pattern had a mean of 0 and standard deviation of 1 . Therefore, the mean univariate signal for each pattern was removed in the ROI.

Decoding accuracies for each subject were computed as the average classification accuracy across train-and-test iterations using a 'leave-one-run-out' cross-validation procedure. This procedure was performed separately for each ROI, trial epoch (Delay and Execute), and pairwise discrimination (left hand vs right hand movements and "Compty" vs "Midwig" in Experiment 1; and hand vs. eye movements in Experiment 2). We statistically assessed decoding significance at the group-level using one-tailed t-tests vs. $50 \%$ chance decoding. To control for the problem of multiple comparisons within each ROI, we applied a Benjamini-Hochberg false-discovery rate (FDR) correction of $q<0.05$.

\section{Searchlight Pattern-Information Analyses}

To complement our MVPA ROI analyses in both Experiments 1 and 2, we also performed a pattern analysis in each subject using the searchlight approach $\stackrel{87}{ }$. Given the scope of this paper (i.e., to examine the top-down modulation of auditory cortex during planning), we constrained this searchlight analysis to the auditory network mask defined by the Intact Speech > Rest contrast using the independent auditory localizer data (allowing us to localize both lower-order and higher-order auditory regions). In this procedure, the SVM classifier moved through each subjects' localizer-defined auditory network in a voxel-by-voxel fashion whereby, at each voxel, a sphere of surrounding voxels (radius of $4 \mathrm{~mm}$; 33 voxels) were extracted, z-scored within pattern (see above), and input into the SVM classifier. The decoding accuracy for that sphere of voxels was then written to the central voxel. This searchlight procedure was performed separately 
with beta coefficient maps for the Delay and Execute epochs based on the GLM procedure described above, which yielded separate Delay and Execute whole-brain decoding maps. To allow for group-level analyses, the decoding maps were smoothed (6mm FWHM Gaussian kernel) in each subject. Then, for each voxel, we assessed statistical significance using a one-tailed t-test versus $50 \%$ chance decoding. Group-level decoding maps for Delay and Execute epochs were thresholded at $p<.01$ and cluster corrected to $p<.05$ using Monte-Carlo style permutation tests with AFNI's 3dClustSim algorithm $\stackrel{88,89}{ }$. 


\section{Acknowledgements}

This work was supported by operating grants from the Canadian Institutes of Health Research (CIHR) awarded to J.R.F. and J.P.G. (MOP126158). J.P.G. was supported by a Natural Sciences and Engineering Research Council (NSERC) Discovery Grant, as well as funding from the Canadian Foundation for Innovation. D.J.G. was supported by a R.S. McLaughlin Fellowship and an NSERC graduate fellowship; C.N.A. was supported by an NSERC graduate fellowship. The authors would like to thank Martin York, Sean Hickman, and Don O'Brien for technical assistance.

\section{Author Contributions}

J.R.F., I.S.J and J.P.G. designed the experiments. D.J.G. and J.P.G. performed research. D.J.G., C.N.A., C.H., J.R.F and J.P.G., analyzed and interpreted data. D.J.G., J.R.F and J.P.G wrote the paper. All authors provided edits and feedback on the final version of the paper.

\section{Competing Interests Statement}

The authors declare no competing financial interests. 


\section{REFERENCES}

1. Tanji, J. \& Evarts, E. V. Anticipatory activity of motor cortex neurons in relation to direction of an intended movement. J. Neurophysiol. 39, 1062-1068 (1976).

2. Messier, J. \& Kalaska, J. F. Covariation of primate dorsal premotor cell activity with direction and amplitude during a memorized-delay reaching task. J. Neurophysiol. 84, 152-165 (2000).

3. Churchland, M. M. et al. Neural population dynamics during reaching. Nature 487, 51-56 (2012).

4. Wolpert, D. M. \& Flanagan, J. R. Motor prediction. Current Biology vol. 11 R729-R732 (2001).

5. Flanagan, J. R., Bowman, M. C. \& Johansson, R. S. Control strategies in object manipulation tasks. Curr. Opin. Neurobiol. 16, 650-659 (2006).

6. Johansson \& Flanagan, J. R. Coding and use of tactile signals from the fingertips in object manipulation tasks. Nat. Rev. Neurosci. 10, 345-359 (2009).

7. Safstrom, D., Johansson, R. S. \& Flanagan, J. R. Gaze behavior when learning to link sequential action phases in a manual task. Journal of Vision vol. 14 3-3 (2014).

8. Schneider, D. M. \& Mooney, R. How Movement Modulates Hearing. Annual Review of Neuroscience vol. 41 553-572 (2018).

9. Hackett, T. A. Anatomic organization of the auditory cortex. Handb. Clin. Neurol. 129, 27-53 (2015).

10. Kumar, S. et al. A Brain System for Auditory Working Memory. Journal of Neuroscience vol. 36 4492-4505 (2016).

11. Linke, A. C. \& Cusack, R. Flexible information coding in human auditory cortex during perception, imagery, and STM of complex sounds. J. Cogn. Neurosci. 27, 1322-1333 (2015).

12. Nelson, A. et al. A circuit for motor cortical modulation of auditory cortical activity. J. Neurosci. 33, 14342-14353 (2013).

13. Schneider, D. M., Nelson, A. \& Mooney, R. A synaptic and circuit basis for corollary discharge in the auditory cortex. Nature 513, 189-194 (2014).

14. Schneider, D. M., Sundararajan, J. \& Mooney, R. A cortical filter that learns to suppress the acoustic consequences of movement. Nature 561, 391-395 (2018).

15. Reznik, D., Ossmy, O. \& Mukamel, R. Enhanced auditory evoked activity to self-generated sounds is mediated by primary and supplementary motor cortices. J. Neurosci. 35 , 2173-2180 (2015).

16. Poeppel, D., Overath, T., Popper, A. N. \& Fay, R. R. The Human Auditory Cortex. (Springer Science \& Business Media, 2012).

17. Werner-Reiss, U., Kelly, K. A., Trause, A. S., Underhill, A. M. \& Groh, J. M. Eye Position Affects Activity in Primary Auditory Cortex of Primates. Current Biology vol. 13 554-562 (2003).

18. Gallivan, J. P., McLean, D. A., Flanagan, J. R. \& Culham, J. C. Where one hand meets the other: limb-specific and action-dependent movement plans decoded from preparatory signals in single human frontoparietal brain areas. J. Neurosci. 33, 1991-2008 (2013).

19. Cisek, P., Crammond, D. J. \& Kalaska, J. F. Neural activity in primary motor and dorsal 
premotor cortex in reaching tasks with the contralateral versus ipsilateral arm. $J$. Neurophysiol. 89, 922-942 (2003).

20. Weinrich, M., Wise, S. P. \& Mauritz, K. H. A neurophysiological study of the premotor cortex in the rhesus monkey. Brain 107 ( Pt 2), 385-414 (1984).

21. Lara, Elsayed, G. F., Zimnik, A. J., Cunningham, J. P. \& Churchland, M. M. Conservation of preparatory neural events in monkey motor cortex regardless of how movement is initiated. Elife 7, (2018).

22. Kaufman, M. T. et al. Roles of monkey premotor neuron classes in movement preparation and execution. J. Neurophysiol. 104, 799-810 (2010).

23. Huang, Y., Heil, P. \& Brosch, M. Associations between sounds and actions in early auditory cortex of nonhuman primates. Elife 8, (2019).

24. Porter, R. \& Lemon, R. Corticospinal Function and Voluntary Movement. (Clarendon Press, 1995).

25. Nelson, A. et al. A Circuit for Motor Cortical Modulation of Auditory Cortical Activity. Journal of Neuroscience vol. 33 14342-14353 (2013).

26. Snyder, L. H., Batista, A. P. \& Andersen, R. A. Coding of intention in the posterior parietal cortex. Nature 386, 167-170 (1997).

27. Cui, H. \& Andersen, R. A. Posterior parietal cortex encodes autonomously selected motor plans. Neuron 56, 552-559 (2007).

28. Gallivan, J. P., McLean, D. A., Smith, F. W. \& Culham, J. C. Decoding effector-dependent and effector-independent movement intentions from human parieto-frontal brain activity. $J$. Neurosci. 31, 17149-17168 (2011).

29. Messier, J. \& Kalaska, J. F. Covariation of primate dorsal premotor cell activity with direction and amplitude during a memorized-delay reaching task. J. Neurophysiol. 84, 152-165 (2000).

30. Riehle, A. \& Requin, J. Monkey primary motor and premotor cortex: single-cell activity related to prior information about direction and extent of an intended movement. $J$. Neurophysiol. 61, 534-549 (1989).

31. Churchland, M. M., Santhanam, G. \& Shenoy, K. V. Preparatory activity in premotor and motor cortex reflects the speed of the upcoming reach. J. Neurophysiol. 96, 3130-3146 (2006).

32. Shen, L. \& Alexander, G. E. Preferential representation of instructed target location versus limb trajectory in dorsal premotor area. J. Neurophysiol. 77, 1195-1212 (1997).

33. Pesaran, B., Nelson, M. J. \& Andersen, R. A. Dorsal premotor neurons encode the relative position of the hand, eye, and goal during reach planning. Neuron 51, 125-134 (2006).

34. Batista, A. P. et al. Reference Frames for Reach Planning in Macaque Dorsal Premotor Cortex. Journal of Neurophysiology vol. 98 966-983 (2007).

35. Hocherman, S. \& Wise, S. P. Effects of hand movement path on motor cortical activity in awake, behaving rhesus monkeys. Exp. Brain Res. 83, 285-302 (1991).

36. Churchland, M. M., Cunningham, J. P., Kaufman, M. T., Ryu, S. I. \& Shenoy, K. V. Cortical preparatory activity: representation of movement or first cog in a dynamical machine? Neuron 68, 387-400 (2010).

37. Shenoy, K. V., Sahani, M. \& Churchland, M. M. Cortical control of arm movements: a dynamical systems perspective. Annu. Rev. Neurosci. 36, 337-359 (2013).

38. Pandarinath, C. et al. High performance communication by people with paralysis using an intracortical brain-computer interface. Elife 6, (2017).

39. Sussillo, D., Churchland, M. M., Kaufman, M. T. \& Shenoy, K. V. A neural network that finds a naturalistic solution for the production of muscle activity. Nat. Neurosci. 18, 1025-1033 
(2015).

40. Shenoy, K. V., Sahani, M. \& Churchland, M. M. Cortical control of arm movements: a dynamical systems perspective. Annu. Rev. Neurosci. 36, 337-359 (2013).

41. Wolpert, D. M., Diedrichsen, J. \& Flanagan, J. R. Principles of sensorimotor learning. Nat. Rev. Neurosci. 12, 739-751 (2011).

42. Schneider, D. M. \& Mooney, R. How Movement Modulates Hearing. Annu. Rev. Neurosci. 41, 553-572 (2018).

43. Holst, E. von, von Holst, E. \& Mittelstaedt, H. Das Reafferenzprinzip. Naturwissenschaften vol. 37 464-476 (1950).

44. Crapse, T. B. \& Sommer, M. A. Corollary discharge across the animal kingdom. Nat. Rev. Neurosci. 9, 587-600 (2008).

45. Scott, S. H. The computational and neural basis of voluntary motor control and planning. Trends Cogn. Sci. 16, 541-549 (2012).

46. Scott, S. H. A Functional Taxonomy of Bottom-Up Sensory Feedback Processing for Motor Actions. Trends Neurosci. 39, 512-526 (2016).

47. Jenmalm, P., Schmitz, C., Forssberg, H. \& Ehrsson, H. H. Lighter or heavier than predicted: neural correlates of corrective mechanisms during erroneously programmed lifts. $J$. Neurosci. 26, 9015-9021 (2006).

48. Desmurget, M. et al. Role of the posterior parietal cortex in updating reaching movements to a visual target. Nat. Neurosci. 2, 563-567 (1999).

49. Tunik, E., Frey, S. H. \& Grafton, S. T. Virtual lesions of the anterior intraparietal area disrupt goal-dependent on-line adjustments of grasp. Nat. Neurosci. 8, 505-511 (2005).

50. Otazu, G. H., Tai, L.-H., Yang, Y. \& Zador, A. M. Engaging in an auditory task suppresses responses in auditory cortex. Nat. Neurosci. 12, 646-654 (2009).

51. Schneider, D. M., Nelson, A. \& Mooney, R. A synaptic and circuit basis for corollary discharge in the auditory cortex. Nature vol. 513 189-194 (2014).

52. Foxe, J. J. et al. Auditory-somatosensory multisensory processing in auditory association cortex: an fMRI study. J. Neurophysiol. 88, 540-543 (2002).

53. Schürmann, M., Caetano, G., Hlushchuk, Y., Jousmäki, V. \& Hari, R. Touch activates human auditory cortex. Neuroimage 30, 1325-1331 (2006).

54. Lakatos, P., Chen, C.-M., O’Connell, M. N., Mills, A. \& Schroeder, C. E. Neuronal oscillations and multisensory interaction in primary auditory cortex. Neuron 53, 279-292 (2007).

55. Kayser, C., Petkov, C. I., Augath, M. \& Logothetis, N. K. Integration of touch and sound in auditory cortex. Neuron 48, 373-384 (2005).

56. Gallivan, J. P., Chapman, C. S., Gale, D. J., Flanagan, J. R. \& Culham, J. C. Selective Modulation of Early Visual Cortical Activity by Movement Intention. Cereb. Cortex (2019) doi:10.1093/cercor/bhy345.

57. Gutteling, T. P. et al. Action preparation shapes processing in early visual cortex. J. Neurosci. 35, 6472-6480 (2015).

58. Chapman, C. S., Gallivan, J. P., Culham, J. C. \& Goodale, M. A. Mental blocks: fMRI reveals top-down modulation of early visual cortex when obstacles interfere with grasp planning. Neuropsychologia 49, 1703-1717 (2011).

59. Steinmetz, N. A. \& Moore, T. Eye Movement Preparation Modulates Neuronal Responses in Area V4 When Dissociated from Attentional Demands. Neuron vol. 83 496-506 (2014).

60. Matyas, F. et al. Motor control by sensory cortex. Science 330, 1240-1243 (2010).

61. Scheich, H., Brechmann, A., Brosch, M., Budinger, E. \& Ohl, F. W. The cognitive auditory cortex: task-specificity of stimulus representations. Hear. Res. 229, 213-224 (2007). 
62. Weinberger, N. M. Reconceptualizing the Primary Auditory Cortex: Learning, Memory and Specific Plasticity. The Auditory Cortex 465-491 (2011)

doi:10.1007/978-1-4419-0074-6_22.

63. Oldfield, R. C. The assessment and analysis of handedness: the Edinburgh inventory. Neuropsychologia 9, 97-113 (1971).

64. Gallivan, J. P., Johnsrude, I. S. \& Flanagan, J. R. Planning Ahead: Object-Directed Sequential Actions Decoded from Human Frontoparietal and Occipitotemporal Networks. Cereb. Cortex 26, 708-730 (2016).

65. Gallivan, J. P., Cant, J. S., Goodale, M. A. \& Flanagan, J. R. Representation of object weight in human ventral visual cortex. Curr. Biol. 24, 1866-1873 (2014).

66. Esteban, O. et al. fMRIPrep: a robust preprocessing pipeline for functional MRI. Nat. Methods 1 (2018).

67. Gorgolewski, K. et al. Nipype: a flexible, lightweight and extensible neuroimaging data processing framework in python. Front. Neuroinform. 5, 13 (2011).

68. Esteban, O. et al. nipy/nipype: 1.4.0. (2019). doi:10.5281/zenodo.3588470.

69. Tustison, N. J. et al. N4ITK: improved N3 bias correction. IEEE Trans. Med. Imaging 29, 1310-1320 (2010).

70. Avants, B. B., Epstein, C. L., Grossman, M. \& Gee, J. C. Symmetric diffeomorphic image registration with cross-correlation: evaluating automated labeling of elderly and neurodegenerative brain. Med. Image Anal. 12, 26-41 (2008).

71. Zhang, Y., Brady, M. \& Smith, S. Segmentation of brain MR images through a hidden Markov random field model and the expectation-maximization algorithm. IEEE Trans. Med. Imaging 20, 45-57 (2001).

72. Dale, A. M., Fischl, B. \& Sereno, M. I. Cortical surface-based analysis. I. Segmentation and surface reconstruction. Neuroimage 9, 179-194 (1999).

73. Klein, A. et al. Mindboggling morphometry of human brains. PLoS Comput. Biol. 13, e1005350 (2017).

74. Evans, A. C., Janke, A. L., Collins, D. L. \& Baillet, S. Brain templates and atlases. Neuroimage 62, 911-922 (2012).

75. Greve, D. N. \& Fischl, B. Accurate and robust brain image alignment using boundary-based registration. Neuroimage 48, 63-72 (2009).

76. Jenkinson, M., Bannister, P., Brady, M. \& Smith, S. Improved optimization for the robust and accurate linear registration and motion correction of brain images. Neuroimage 17, 825-841 (2002).

77. Cox, R. W. \& Hyde, J. S. Software tools for analysis and visualization of fMRI data. NMR Biomed. 10, 171-178 (1997).

78. Lanczos, C. Evaluation of Noisy Data. Journal of the Society for Industrial and Applied Mathematics Series B Numerical Analysis 1, 76-85 (1964).

79. Abraham, A. et al. Machine learning for neuroimaging with scikit-learn. Front. Neuroinform. 8, 14 (2014).

80. Mumford, J. A., Turner, B. O., Ashby, F. G. \& Poldrack, R. A. Deconvolving BOLD activation in event-related designs for multivoxel pattern classification analyses.

Neuroimage 59, 2636-2643 (2012).

81. Morosan, P. et al. Population maps of cytoarchitectonically defined human auditory areas. Neurolmage vol. 11 S304 (2000).

82. Da Costa, S. et al. Human primary auditory cortex follows the shape of Heschl's gyrus. J. Neurosci. 31, 14067-14075 (2011).

83. Morosan, P. et al. Human primary auditory cortex: cytoarchitectonic subdivisions and 
mapping into a spatial reference system. Neuroimage 13, 684-701 (2001).

84. Rademacher, J. et al. Probabilistic mapping and volume measurement of human primary auditory cortex. Neuroimage 13, 669-683 (2001).

85. Desikan, R. S. et al. An automated labeling system for subdividing the human cerebral cortex on MRI scans into gyral based regions of interest. Neuroimage 31, 968-980 (2006).

86. Hackett, T. A. et al. Feedforward and feedback projections of caudal belt and parabelt areas of auditory cortex: refining the hierarchical model. Front. Neurosci. 8, 72 (2014).

87. Kriegeskorte, N., Goebel, R. \& Bandettini, P. Information-based functional brain mapping. Proc. Natl. Acad. Sci. U. S. A. 103, 3863-3868 (2006).

88. Cox, R. W. AFNI: software for analysis and visualization of functional magnetic resonance neuroimages. Comput. Biomed. Res. 29, 162-173 (1996).

89. Cox, R. W., Chen, G., Glen, D. R., Reynolds, R. C. \& Taylor, P. A. FMRI Clustering in AFNI: False-Positive Rates Redux. Brain Connect. 7, 152-171 (2017). 